\title{
Assessment of stochastic models for GPS measurements with different types of receivers
}

\author{
LI BoFeng ${ }^{1 \dagger}$, SHEN YunZhong ${ }^{1,2} \&$ XU PeiLiang ${ }^{3}$ \\ ${ }^{1}$ Department of Surveying and Geo-informatics Engineering, Tongji University, Shanghai 200092, China; \\ ${ }^{2}$ Key Laboratory of Advanced Surveying Engineering of SBSM, Shanghai 200092, China; \\ ${ }^{3}$ Disaster Prevention Research Institute, Kyoto University, Uji, Kyoto 611-0011, Japan
}

The stochastic model plays an important role in parameter estimation. The optimal estimator in the sense of least squares can only be obtained by using the correct stochastic model and consequently guarantees the precise positioning in GPS applications. In this contribution, the GPS measurements, collected by different types of geodetic dual-frequency receiver pairs on ultra-short baselines with a sampling interval of $1 \mathrm{~s}$, are used to address their stochastic models, which include the variances of all observation types, the relationship between the observation accuracy and its elevation angle, the time correlation, as well as the correlation between observation types. The results show that the commonly used stochastic model with the assumption that all the raw GPS measurements are independent with the same variance does not meet the need for precise positioning and the elevation-dependent weight model cannot work well for different receiver and observation types. The time correlation and cross correlation are significant as well. It is therefore concluded that the stochastic model is much associated with the receiver and observation types and should be specified for the receiver and observation types.

GPS, stochastic model, elevation dependent weight, time correlation, cross correlation

As an important part in a linear (linearized) observation model, the stochastic model describes the accuracy of observation (variance) and the correlation characteristics (covariance) among observations. If the stochastic model is unreliably determined, the parameter estimates and their accuracies can be disturbed. Therefore, the variance-covariance component estimation (VCE) has been extensively investigated recently and many feasible and simplified algorithms have been proposed for the well- or the ill-conditioned linear model on the basis of minimum variance estimation, least squares estimation, maximum likelihood estimation, minimum norm quadratic unbiased estimation and best invariable quadratic unbiased estimation ${ }^{[1-8]}$. $\mathrm{Xu}$ et al. ${ }^{[5]}$ proposed the estimability concept of VCE and proved that at most $r(r+1) / 2$ independent variance-covariance components are estimable with redundancy $r$.
The precise positioning can be carried out only in the case of the known adequate stochastic model of GPS measurements and, therefore the significant research efforts have been made towards stochastic modeling for GPS measurements in the past decade ${ }^{[9-15]}$. The earlier study by Euler and Goad ${ }^{[9]}$ described the elevation-dependent weight method, in which an exponential formula with respect to the elevation angles of the tracked satellites is employed to approximate the accuracies of GPS measurements, and the positioning accuracy is really somewhat improved in real applications ${ }^{[15]}$. Nevertheless, this method does not take the correlation

Received March 7, 2008; accepted May 21, 2008; published online August 27, 2008 doi: 10.1007/s11434-008-0293-6

†Corresponding author (email: Bofeng_Li@163.com)

Supported partially by the National Natural Science Foundation of China (Grant No. 40674003), partially by a Grant-in-Aid for Scientific Research (Grant No. B19340129), and the Project From Science and Technology Commission of Shanghai Municipality (Grant No. 06DZ22101) 
among observations into account. An iterative stochastic assessment procedure by using the MINQUE method, which takes all of the error features into account, has been proposed by Wang et al. ${ }^{[10]}$ to improve the accuracy of baseline. However, it is inconvenient to implement and suitable only for a short observation period because both the time correlation coefficients and the variances of GPS measurements are assumed as constant for the whole observation period in this iterative procedure $^{[10,11,14]}$. Bona and Tiberius ${ }^{[12]}$ and Kenselaar ${ }^{[16]}$ addressed the relationship between the observation accuracy and its elevation angle, the correlation between measurements of the same type, as well as the correlation between observations of different types, namely cross correlation using GPS zero baseline data. He and Yang ${ }^{[17]}$ proposed a method to real-time estimate the prior VCE of GPS double differenced (DD) observables and it is successfully performed in kinematic positioning. In this contribution, the GPS measurements are collected by different types of geodetic dual-frequency receiver pairs on ultra-short baselines with a sampling interval of $1 \mathrm{~s}$ and used to address the variances of all observation types, relationship between the observation accuracy and its elevation angle, time correlation, as well as cross correlation between observation types.

\section{GPS single differenced observation model with a known baseline and integer ambiguity}

\subsection{Reparameterization of GPS single differenced observation model}

In GPS relative positioning, the DD model is favored because it can, to a large extent, eliminate or reduce many systematic errors, e.g., clock errors of receivers and satellites, troposphere delay, ionosphere delay and so on, and guarantee ambiguities with the property of integer. However, the single differenced (SD) model is preferred in case of VCE for GPS measurements with following reasons ${ }^{[12,13,15,16]}$ : (1) it is rather easy to form the SD measurements from raw data without employment of reference satellite; (2) the mathematical correlation is not introduced except that variance of SD observation is enlarged by twice with respect to that of raw one; and (3) it is also easy to assess those stochastic properties assigned to satellites based on SD residuals (e.g., correlation between satellites), because only one satellite is involved in SD observation.

In this contribution, the dual-frequency observations of ultra-short baselines of about $5 \mathrm{~m}$ are used, and as a result, the residual systematic effects are neglected in the SD model. The SD observation equation for L1 phase and $\mathrm{P} 1$ code can be described as

$$
\begin{aligned}
& \nabla P_{L 1}^{s}=\nabla \rho^{s}+\nabla \delta t_{L 1}+\nabla \delta t_{0, L 1}-\lambda_{1} \nabla N_{L 1}^{s}+\nabla \varepsilon_{L 1}^{s}, \\
& \nabla P_{P 1}^{s}=\nabla \rho^{s}+\nabla \delta t_{P 1}+\nabla \delta t_{0, P 1}+\nabla \varepsilon_{P 1}^{s},
\end{aligned}
$$

where " $\nabla$ " indicates the SD operator; $\nabla P_{L 1}^{s}$ and $\nabla P_{P 1}^{s}$ are the SD observables respectively for $\mathrm{L} 1$ phase and $\mathrm{P} 1$ code; $\nabla \rho^{s}$ is the SD satellite-to-receiver distance; $\nabla \delta t_{L 1}$ and $\nabla \delta t_{P 1}$ are receiver clock errors for $\mathrm{L} 1$ phase and P1 code; $\nabla \delta t_{0, L 1}, \nabla \delta t_{0, P 1}, \nabla \varepsilon_{L 1}^{s}$ and $\nabla \varepsilon_{P 1}^{s}$ are hardware delays of receiver and observation noises for L1 phase and P1 code respectively; $\nabla N_{L 1}^{s}$ is SD integer ambiguity for L1 phase; $\lambda_{1}$ is wavelength of L1 phase; the superscript of " $s$ " indicates tracked satellite. Undoubtedly, the observation equations for L2 phase, $\mathrm{C} 1$ and $\mathrm{P} 2$ code can be analogously formulated.

The parameters in eq. (1) are rearranged, namely reparameterization, in order to use the known integer DD ambiguity,

$$
\begin{gathered}
\nabla P_{L 1}^{s}=\nabla \rho^{s}+\nabla \delta t_{L 1}+\nabla \delta t_{0, L 1}-\lambda_{1}\left(\nabla N_{L 1}^{s}-\nabla N_{L 1}^{r}\right. \\
\left.+\nabla N_{L 1}^{r}\right)+\nabla \varepsilon_{L 1}^{s}=\nabla \rho^{s}+\nabla \delta_{L 1}-\lambda_{1} \Delta N_{L 1}^{r s}+\nabla \varepsilon_{L 1}^{s}
\end{gathered}
$$

where $\nabla \delta_{L 1}=\nabla \delta t_{L 1}+\nabla \delta t_{0, L 1}-\lambda_{1} \nabla N_{L 1}^{r}$ is called equivalent receiver clock error for $\mathrm{L} 1$ phase and $\Delta N_{L 1}^{r s}$ is the DD ambiguity with reference satellite " $r$ ". The reparameterized P1 code SD observation equation can also be derived by combining the receiver clock error with receiver hardware delay,

$$
\nabla P_{P 1}^{s}=\nabla \rho^{s}+\nabla \delta_{P 1}+\nabla \varepsilon_{P 1}^{s},
$$

with the equivalent receiver clock error for P1 code $\nabla \delta_{P 1}=\nabla \delta t_{P 1}+\nabla \delta t_{0, P 1}$.

\subsection{GPS single differenced observation equation with a known baseline and integer ambiguity}

Substituting the baseline and DD ambiguities that are preciously determined by using the whole observation series into eq. (2), the SD observation equation with fixed baseline and ambiguities is described as

$$
\begin{aligned}
& \nabla \tilde{P}_{P 1}^{s}=\nabla P_{P 1}^{s}-\nabla \rho^{s}=\nabla \delta_{P 1}+\nabla \varepsilon_{P 1}^{s}, \\
& \nabla \tilde{P}_{L 1}^{s}=\lambda_{1} \nabla \varphi_{L 1}^{s}-\nabla \rho^{s}+\lambda_{1} \Delta N_{L 1}^{r s}=\nabla \delta_{L 1}+\nabla \varepsilon_{L 1}^{s} .
\end{aligned}
$$


Assuming total $m$ satellites are simultaneously tracked, the SD observation equation of $\mathrm{P} 1$ code for one epoch can be symbolized as

$$
\nabla \tilde{\boldsymbol{P}}_{P 1}=\boldsymbol{e}_{m} \nabla \delta_{P 1}+\nabla \boldsymbol{\varepsilon}_{P 1},
$$

where $\quad \nabla \tilde{\boldsymbol{P}}_{P 1}=\left(\begin{array}{lllll}\nabla \tilde{P}_{P 1}^{s_{1}} & \nabla \tilde{P}_{P 1}^{s_{2}} & \cdots & \nabla \tilde{P}_{P 1}^{s_{m}}\end{array}\right)^{T}$ is the column vector of SD observables; $\boldsymbol{e}_{m}=\left(\begin{array}{llll}1 & 1 & \cdots & 1\end{array}\right)_{m \times 1}^{T}$ is design matrix; $\nabla \boldsymbol{\varepsilon}_{P 1}=\left(\begin{array}{lllll}\nabla \varepsilon_{P 1}^{s_{1}} & \nabla \varepsilon_{P 1}^{s_{2}} & \cdots & \nabla \varepsilon_{P 1}^{s_{m}}\end{array}\right)^{T}$ is the SD observation noise vector and the superscript " $s_{i}$ " indicates the $i$ th tracked satellite. The SD observation equations of the other observation types for one epoch can be analogously derived.

\section{Assessment of stochastic model for GPS measurements}

As the integer DD ambiguities are previously fixed, the phase observation is actually converted to virtual pseudorange and, therefore all estimation formulae below are derived only for $\mathrm{P} 1$ code.

\subsection{Accuracy estimation for observation types}

In this procedure, it is assumed that all the raw measurements are independent and their accuracies depend only on the observation types (e.g., P1 code and L1 phase). Therefore, the covariance matrix with respect to (4) can be expressed as

$$
\mathrm{D}\left(\nabla \tilde{\boldsymbol{P}}_{P 1}\right)=\boldsymbol{Q}_{\nabla P 1}=\sigma_{\nabla P 1}^{2} \boldsymbol{I}_{m},
$$

where $\mathrm{D}(\bullet)$ is the variance operator; $\sigma_{\nabla P 1}^{2}$ is the variance of SD P1 code observation; $\boldsymbol{I}_{m}$ is the identity matrix with dimension of $m$. Due to the fact that two receivers of the same type are used and the observation environments for the ultra-short baseline are almost the same, we can adequately assume the same stochastic model for these two receivers,

$$
\boldsymbol{Q}_{\nabla P 1}=2 \boldsymbol{Q}_{P 1},
$$

where $\boldsymbol{Q}_{P 1}$ is covariance matrix of zero differenced (ZD) measurements for one epoch. Therefore, the accuracy of $\mathrm{P} 1$ code can be determined by

$$
\sigma_{P 1}=\sqrt{\frac{\boldsymbol{v}^{T} \boldsymbol{v}}{2(m-1)}}
$$

with $\boldsymbol{v}=\nabla \tilde{\boldsymbol{P}}_{P 1}-\frac{\boldsymbol{e}_{m} \boldsymbol{e}_{m}^{T} \nabla \tilde{\boldsymbol{P}}_{P 1}}{m}$ being the residual vector of P1 measurements.

\subsection{The relation of accuracy and elevation angle}

The measurements of one satellite over several seconds can be adequately assumed to have a constant variance, because of a very small variation of elevation angle over this short period. If the variance remains unchanged for $n$ consecutive epochs, the corresponding stochastic model is given as

$$
\boldsymbol{Q}_{\nabla P 1}^{n}=\boldsymbol{I}_{n} \otimes \boldsymbol{Q}_{\nabla P 1},
$$

where $\boldsymbol{Q}_{\nabla P 1}=\operatorname{diag}\left(\left(\sigma_{\nabla P 1}^{s_{1}}\right)^{2} \quad\left(\sigma_{\nabla P 1}^{s_{2}}\right)^{2} \cdots\left(\sigma_{\nabla P 1}^{s_{m}}\right)^{2}\right)$ is covariance matrix of SD P1 measurements for one epoch with $\operatorname{diag}(\bullet)$ standing for the diagonal matrix from a vector and $\sigma_{\nabla P 1}^{s_{i}}$ the standard deviation of P1 measurement of the $i$ th satellite. " $\otimes$ " is Kronecker product. The accuracy of ZD observation of the $i$ th satellite can be estimated by

$$
\sigma_{P 1}^{s_{i}}=\sqrt{\sum_{j=1}^{n}\left(v_{j}^{i}\right)^{2} / 2 n r_{j}^{i}},
$$

where $v_{j}^{i}=\nabla \tilde{P}_{P 1, j}^{s_{i}}-\sum_{k=1}^{m} \nabla \tilde{P}_{P 1, j}^{s_{k}} / m$ is the residual of SD measurement of the $i$ th satellite at the $j$ th epoch and $r_{j}^{i}=(m-1) / m$ is its redundancy. In terms of estimated accuracy of SD measurement above and its corresponding elevation angle, the relationship between accuracy and elevation angle can be evaluated.

\subsection{Time correlation estimation}

The time correlation of observation series will be addressed and the correlation between measurements from different satellites is still ignored. The covariance matrix of observation series of one satellite with $n$ epochs is described as

$$
\mathrm{D}\left(\begin{array}{c}
\nabla \tilde{\boldsymbol{P}}_{P 1,1} \\
\nabla \tilde{\boldsymbol{P}}_{P 1,2} \\
\vdots \\
\nabla \tilde{\boldsymbol{P}}_{P 1, n}
\end{array}\right)=\left(\begin{array}{cccc}
\sigma_{\nabla P 1,1}^{2} \boldsymbol{I}_{m} & \rho_{12} \sigma_{\nabla P 1,1} \sigma_{\nabla P 1,2} \boldsymbol{I}_{m} & \cdots & \rho_{1 n} \sigma_{\nabla P 1,1} \sigma_{\nabla P 1, n} \boldsymbol{I}_{m} \\
\rho_{21} \sigma_{\nabla P 1,2} \sigma_{\nabla P 1,1} \boldsymbol{I}_{m} & \sigma_{\nabla P 1,2}^{2} \boldsymbol{I}_{m} & \cdots & \rho_{2 n} \sigma_{\nabla P 1,2} \sigma_{\nabla P 1, n} \boldsymbol{I}_{m} \\
\vdots & \cdots & \ddots & \vdots \\
\rho_{n 1} \sigma_{\nabla P 1, n} \sigma_{\nabla P 1,1} \boldsymbol{I}_{m} & \rho_{n 2} \sigma_{\nabla P 1, n} \sigma_{\nabla P 1,2} \boldsymbol{I}_{m} & \cdots & \sigma_{\nabla P 1, n}^{2} \boldsymbol{I}_{m}
\end{array}\right),
$$


where the variance of SD P1 observation at the $i$ th epoch $\sigma_{\nabla P 1, i}^{2}=2 \sigma_{P 1, i}^{2}$ can be calculated by eq. (7); $\rho_{i j}=\rho_{j i}$ is the time correlation coefficient between the $i$ th and $j$ th epochs. The autocorrelation function is an important mathematical tool for describing the correlation of an observation series in time domain, which is defined as

$$
\rho_{\tau}=\rho_{i j}=\rho_{j i}, \quad \tau=|i-j|, \quad(i, j=1, \cdots, n) .
$$

Therefore, the autocorrelation coefficient, the element of autocorrelation function, can be derived as

$$
\rho_{\tau}=\frac{1}{2(n-\tau)} \sum_{i=1}^{n-\tau} \frac{\boldsymbol{v}_{i}^{T} \boldsymbol{v}_{i+\tau}}{\sqrt{r_{i} r_{i+\tau}} \sigma_{P 1, i} \sigma_{P 1, i+\tau}} 1 \leqslant \tau \leqslant n-1,
$$

where $\quad \boldsymbol{v}_{i}=\nabla \tilde{\boldsymbol{P}}_{P 1, i}-\boldsymbol{e}_{m} \sum_{j=1}^{m} \nabla \tilde{P}_{P 1, i}^{s_{j}} / m \quad$ is the residual vector at the $i$ th epoch; $\sigma_{P 1, i}$ and $\sigma_{P 1, i+\tau}$ are the standard deviations of ZD observation at the $i$ th and $(i+\tau)$ th epochs respectively; $r_{i}$ and $r_{i+\tau}$ are the redundancies of SD observation vector at the $i$ th epoch and the $(i+\tau)$ th epoch and they are satisfied with $r_{i}=r_{i+\tau}$ $=m-1$.

\subsection{Cross correlation estimation}

The cross correlation between observations of different types is studied here. Considering two observation types, e.g., P1 and P2, the stochastic model of single epoch reads

$$
\mathrm{D}\left(\begin{array}{c}
\nabla \tilde{\boldsymbol{P}}_{P 1} \\
\nabla \tilde{\boldsymbol{P}}_{P 2}
\end{array}\right)=\left(\begin{array}{cc}
\sigma_{\nabla P 1}^{2} \boldsymbol{I}_{m} & \rho_{P 1, P 2} \sigma_{\nabla P 1} \sigma_{\nabla P 2} \boldsymbol{I}_{m} \\
\rho_{P 2, P 1} \sigma_{\nabla P 2} \sigma_{\nabla P 1} \boldsymbol{I}_{m} & \sigma_{\nabla P 2}^{2} \boldsymbol{I}_{m}
\end{array}\right),
$$

where $\sigma_{\nabla P 1}$ and $\sigma_{\nabla P 2}$ are the accuracies of SD P1 and SD P2 which can be previously determined by eq. (7); the cross correlation coefficients $\rho_{P 1, P 2}=\rho_{P 2, P 1}$ can be calculated by

$$
\rho_{P 1, P 2}=\frac{\boldsymbol{v}_{P 1}^{T} \boldsymbol{v}_{P 2}}{2 \sqrt{r_{P 1} r_{P 2}} \sigma_{P 1} \sigma_{P 2}},
$$

where $\quad \boldsymbol{v}_{P 1}=\nabla \tilde{\boldsymbol{P}}_{P 1}^{s_{i}}-\boldsymbol{e}_{m} \sum_{l=1}^{m} \nabla \tilde{P}_{P 1}^{s_{l}} / m \quad$ and $\quad \boldsymbol{v}_{P 2}=\nabla \tilde{\boldsymbol{P}}_{P 2}^{s_{i}}$ $-\boldsymbol{e}_{m} \sum_{l=1}^{m} \nabla \tilde{P}_{P 2}^{s_{l}} / m$ are the residual vectors with respect to SD P1 and P2 observables of single epoch, and $r_{P 1}$ and $r_{P 2}$ are their redundancies with $r_{P 1}=r_{P 2}=m-1$. The cross correlation coefficients between the other observation types can be analogously estimated.

\section{Experiments and analysis}

The GPS data sets collected with the different types of receivers are implemented according to the formulae derived above. However, only the results for Ashtech Z12 and DNSP receivers are presented in this contribution. The detailed information of data sets is illustrated in Table 1. As mentioned in Sect. 1, the baseline and DD integer ambiguities are previously determined by using the whole observation series to form the constrained SD observation equation and assess stochastic properties of GPS measurements. It is emphasized that 18500 and 5500 epoch's data respectively are used to resolve ultra-short baselines with lengths of about $5 \mathrm{~m}$ and, therefore the achieved baselines are so precise that their influence can be basically ignored.

The accuracy series of ZD measurements for all observation types of two receivers are first calculated epoch by epoch according to eq. (7), and the final estimators are obtained by averaging these epochwise estimators, see e.g., the diagonal elements of Tables 2 and 3. The results have shown that the accuracies of $\mathrm{C} 1, \mathrm{P} 1$ and P2 for Ashtech Z12 are rather close and smaller than $0.25 \mathrm{~m}$. However, the accuracies of P1 and P2 for DNSP are obviously better than that of $\mathrm{C} 1$, and the accuracies of all code observation types for DNSP are worse than that of Ashtech Z12. It should be noticed that the accuracy of $\mathrm{C} / \mathrm{A}$ code is slightly better than that of $\mathrm{P}$ code for Ashtech Z12. This may be why the decoded P code has a lower accuracy than its theoretical value by employing the $\mathrm{Z}$ tracking technique, and additionally the filtering technique is also used to smooth different types of code observations to different extent ${ }^{[18]}$. It is also indicated that the accuracies of L1 and L2 for Ashtech Z12 and L1 for DNSP are smaller than $4 \mathrm{~mm}$, and the L1 accuracy of $2.8 \mathrm{~mm}$ for DNSP is significantly better than that of L2 (7.2 mm).

In order to analyze the relationship between the observation accuracies and their elevation angles, the observation accuracies of all observation types are estimated over each 10 epochs by eq. (9) and the corresponding elevation angles are recorded as well. In the estimated accuracy series, the multiple estimators are always assigned to the same elevation angle and the final estimator with respect to this elevation angle is obtained by averaging these estimators. The relationships between observation accuracies and elevation angles are illustrated respectively for all observation types of 
Ashtech Z12 and DNSP in Figures 1 and 2. For Ashtech $\mathrm{Z} 12$, the modest elevation dependence is present for $\mathrm{C} 1$, P1, P2 and L1 (a factor of about 3 between the lowest accuracy with elevation angle of about $10^{\circ}$ and the highest with about $80^{\circ}$ elevation angle), whereas a strong elevation dependence is really existent for L2 (the factor is about 7). For DNSP, observation types of C1, P1, P2 and L1 do show the strong elevation dependence, but L2 measurements show no elevation dependence at all. Therefore, the following remarks are given: (1) the elevation dependence is subject to the receiver and observation type; and (2) the commonly used elevationdependent weight function does not work well for all receiver and observation types. It should be refined according to the receiver and observation type.

In fact, the filtering technique is employed by many

Table 1 GPS data sets for Ashtech Z12 and DNSP receivers

\begin{tabular}{|c|c|c|c|c|c|}
\hline Receiver & Observation types & Length of baseline (m) & Sample interval (s) & Mask elevation angle $\left({ }^{\circ}\right)$ & Number of epochs \\
\hline Ashtech Z12 & $\mathrm{C} 1 / \mathrm{P} 1 / \mathrm{P} 2 / \mathrm{L} 1 / \mathrm{L} 2$ & 4.705 & 1 & 10 & 18500 \\
\hline DSNP & $\mathrm{C} 1 / \mathrm{P} 1 / \mathrm{P} 2 / \mathrm{L} 1 / \mathrm{L} 2$ & 6.446 & 1 & 10 & 5500 \\
\hline
\end{tabular}
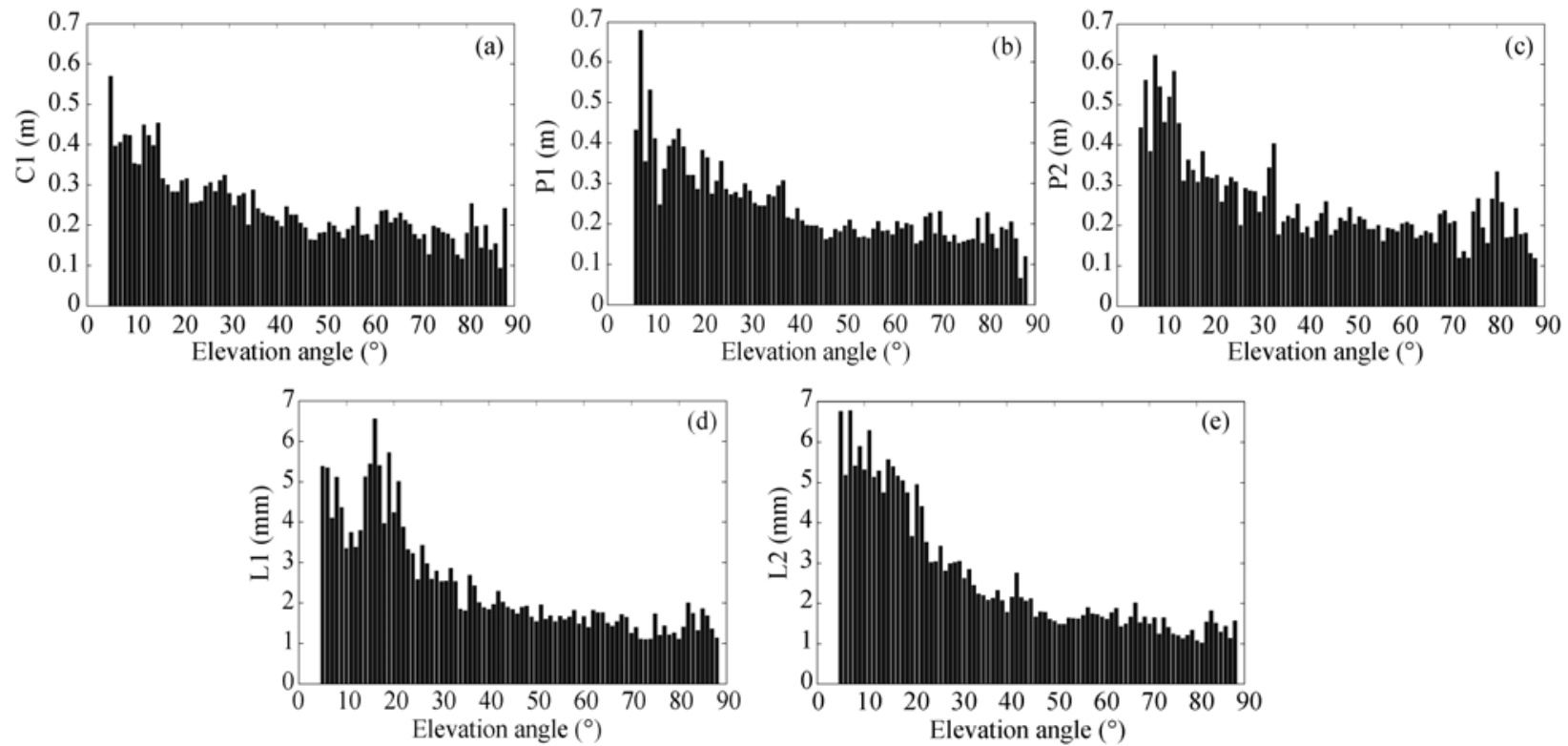

Figure 1 The relationship of accuracy and elevation angle for Ashtech receiver.
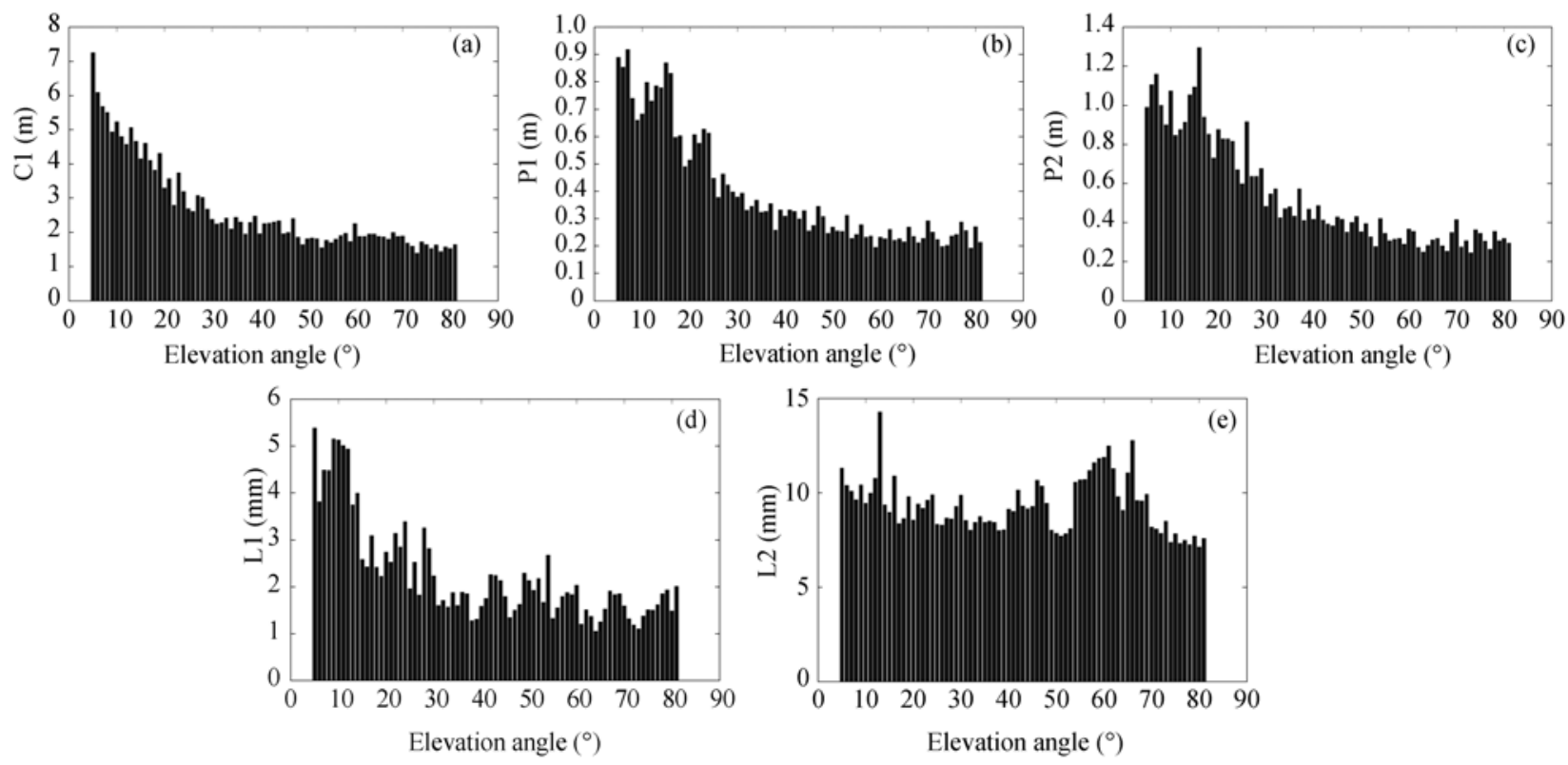

Figure 2 The relationship of accuracy and elevation angle for DNSP receiver. 
types of receivers to improve the observation accuracy and it consequentially leads to the time correlation. The automatic correlation coefficients are estimated by eq. (12) for all observation types of Ashtech Z12 and DNSP, e.g. see Figures 3 and 4. For Ashtech Z12, the time correlation is significant for all observation types and the correlation coefficients are still larger than 0.5 at lag of $50 \mathrm{~s}$. For DNSP, a modest time correlation is present for $\mathrm{C} 1$ with the lag of only $6 \mathrm{~s}$ and strong time correction for the other observation types and the correlation coefficients for P1 and P2 decrease to 0.2 after about $30 \mathrm{~s}$. The time correlation coefficient for L1 phase of DNSP decreases very slowly and is still larger than 0.5 at a lag of $50 \mathrm{~s}$. It is, however, relatively stable to about 0.2 when the lag is larger than $50 \mathrm{~s}$. The time correlation
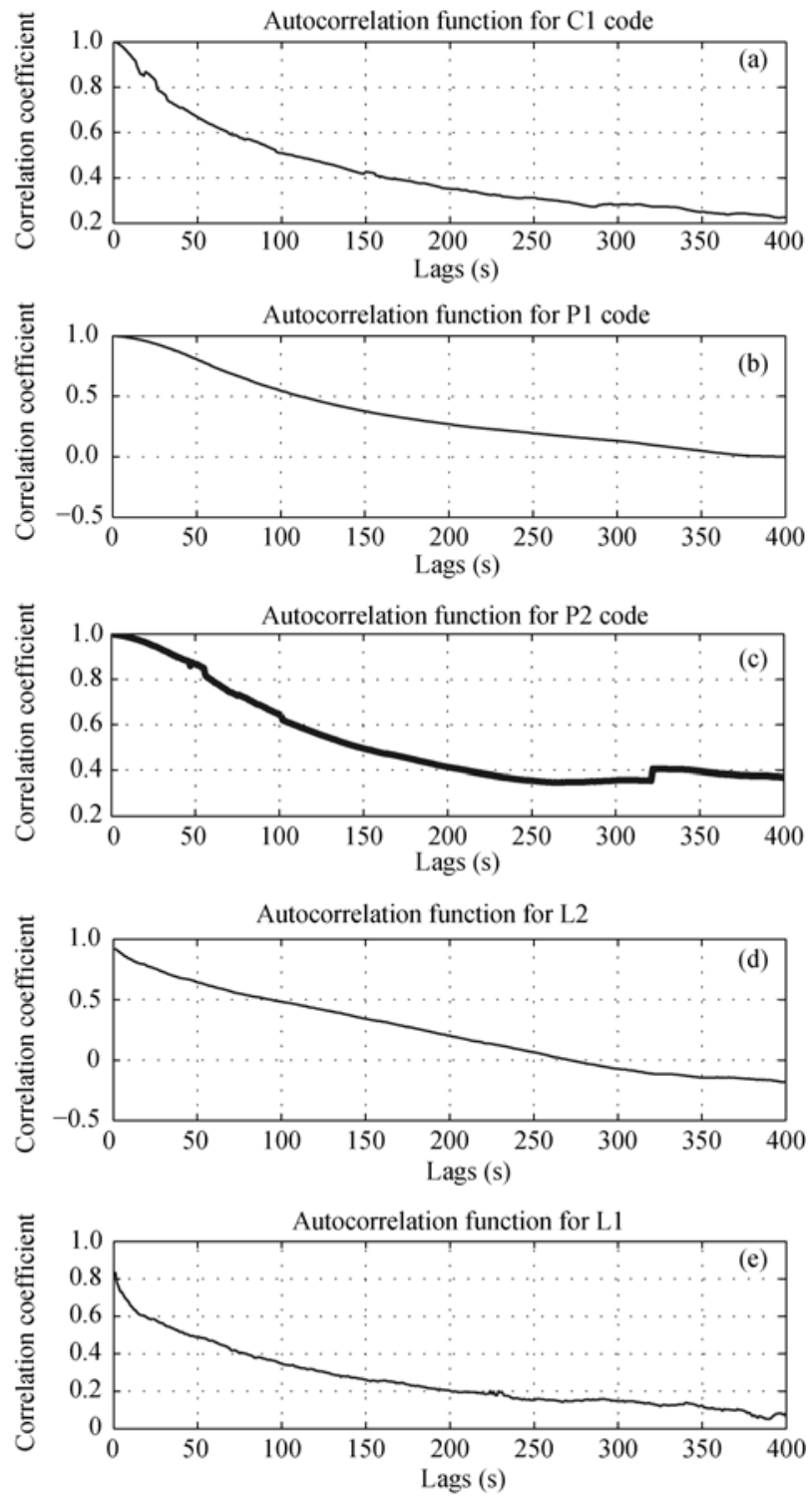

Figure 3 The time correlation for Ashtech receiver. coefficient for DNSP L2 phase is about 0.35 at a lag of 1 s. Considering the time correlation and observation accuracy (see Figures 3 and 4, and Tables 2 and 3), it seems that the higher observation accuracy is always associated to the longer lag of time correlation. In other words, the higher observation accuracy is implemented by a longer time filtering ${ }^{[12]}$. For instance, $\mathrm{C} 1$ accuracy of Ashtech Z12 is higher than that of DNSP because of the longer time filtering for Ashtech Z12 C1 code. Anyway, the time correlation for some observation types of some receivers is very significant and should be taken into account for precise GPS positioning

In addition, the cross correlation can be introduced by some decoding techniques employed by some types of receivers. For example, when the code correlation tech-
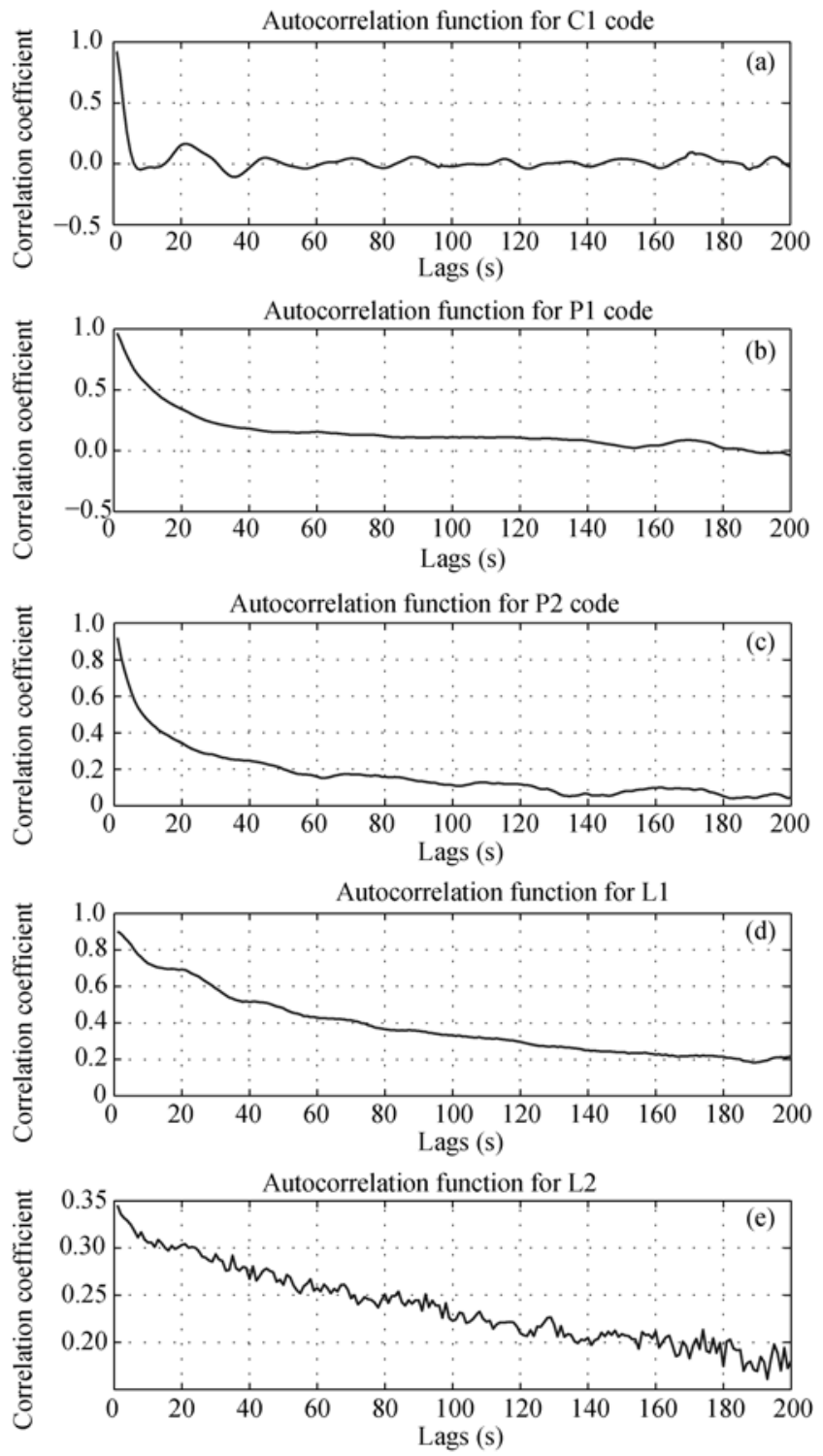

Figure 4 The time correlation for DNSP receiver. 
Table 2 The accuracy and cross correlation for Ashtech receiver (unit: code $(\mathrm{m}) \&$ phase $(\mathrm{mm})$

\begin{tabular}{cccccc}
\hline & C1 & P1 & P2 & L1 & L2 \\
\hline C1 & 0.229 & 0.536 & 0.014 & -0.028 & -0.028 \\
P1 & & 0.244 & 0.077 & 0.004 & -0.004 \\
P2 & & & 0.233 & -0.012 & -0.063 \\
L1 & & & & 3.3 & 0.233 \\
L2 & & & & & 2.3 \\
\hline
\end{tabular}

Table 3 The accuracy and cross correlation for DSNP receiver (unit: code $(\mathrm{m}) \&$ phase $(\mathrm{mm})$

\begin{tabular}{cccccc}
\hline & C1 & P1 & P2 & L1 & L2 \\
\hline C1 & 3.161 & 0.124 & 0.017 & 0.029 & 0.002 \\
P1 & & 0.468 & 0.119 & 0.021 & -0.017 \\
P2 & & & 0.645 & -0.045 & 0.053 \\
L1 & & & & 2.8 & -0.001 \\
L2 & & & & & 7.2 \\
\hline
\end{tabular}

nique is used to acquire the $\mathrm{P}$ code which is encrypted under Anti-Spoofing, the $\mathrm{C} 1$ code and $\Delta \mathrm{P}=\mathrm{P} 1-\mathrm{C} 1$ are directly acquired rather than $\mathrm{C} 1$ code and $\mathrm{P} 1$ code, and the $\mathrm{P} 1$ code is determined by $\mathrm{P} 1=\Delta \mathrm{P}+\mathrm{C} 1$. Consequently, it leads to the cross correlation between $\mathrm{C} 1$ code and $\mathrm{P} 1$ code. The cross correlation between any two observation types of Ashtech Z12 and DNSP are computed by eq. (14) and the results are presented in Tables 2 and 3 (see e.g., the off-diagonal elements). For Ashtech Z12, C1 code is strongly correlated with P1 code with the correlation coefficient of 0.536 , and modest correlation for L1 and L2 with coefficient of 0.233 and the correlation between other observation types seems nonexistent. However, only the feeble correlation is existent both between $\mathrm{C} 1$ and P1 and between P1 and P2 for DNSP. The same

1 Yu Z C. A universal formulae of maximum likelihood estimation for variance-covariance estimation. J Geod, 1996, 70: 233-240

2 Crocetto N, Gatti M, Russo P. Simplified formulae for the BIQUE estimation of variance components in disjunctive observation group. $\mathrm{J}$ Geod, 2000, 74: 447-457

3 Grodecki J. Generalized maximum-likelihood estimation of variance-covariance components with non-informative prior. J Geod, 2001, 75: 157-163

4 Teunissen P, Amiri-Simkooei A. Least-squares variance component estimation. J Geod, 2007, 82: 65-82

5 Xu P L, Liu Y M, Shen Y Z, et al. Estimability analysis of variance and covariance components. J Geod, 2007, 81: 593-602

6 Ananga N, Coleman R, Rizos C. Variance-covariance estimation of GPS networks. Bull Geod, 1994, 68: 77-87

7 Koch K, Kusche J. Regularization of geopotential determination from satellite data by variance components. J Geod, 2002, 76: 259-268

8 Xu P L, Shen Y Z, Fukuda Y, et al. Variance component estimation in linear inverse ill-posed models. J Geod, 2006, 80: 69-81

9 Euler H, Goad C. On optimal filtering of GPS dual frequency observations without using orbit information. Bull Geod, 1991, 65: $130-143$ applies to other observation types.

\section{Conclusions}

The formulae for assessing the stochastic properties of GPS measurements are derived based on the SD observation equation with a precisely known baseline and fixed DD integer ambiguities. GPS data sets are collected with different types of dual-frequency receivers in ultra-short baseline scenarios and are used to demonstrate their stochastic properties. The results from different receivers are analyzed and the remarking conclusions are summarized.

(1) The commonly used elevation-dependent weight function does not work well for all observation types of any receiver and it should be refined by the observation type of receiver.

(2) The filtering technique in time domain can improve the observation accuracy, while the time correlation is introduced. Therefore, it should be taken into account, especially the lag of time correlation, for precision GPS application.

(3) The cross correlation can also, to a certain extent, be introduced by decoding techniques of some types of receivers and should be addressed in real application.

On all accounts, the elevation dependence, time correlation, as well as cross correlation between observation types are existent in GPS observation series. Therefore, the stochastic model should be adequately refined according to observation types of receiver in order to achieve the precision GPS positioning.

10 Wang J, Stewart M, Sakiri M. Stochastic modeling for static GPS baseline data processing. J Surv Eng, 1998, 124(4): 171-181

11 Satirapod C, Wang J, Rizos C. A new stochastic modelling procedure for precise static GPS positioning. ZFV, 2001, 126(6): 365-373

12 Bona P. Precision, cross correlation, and time correlation of GPS phase and code observations. GPS Solut, 2000, 4(2): 3-13

13 Tiberius C, Kenselaar F. Estimation of the stochastic model for GPS code and phase observation. Surv Rev, 2000, 35(277): 441-454

14 Wang J, Satirapod C, Rizos C. Stochastic assessment of GPS carrier phase measurements for precise static relative positioning. J Geod, 2002, 76: $95-104$

15 Liu X L. A comparison of stochastic models for GPS single differential kinematic positioning. In: Procceedings of ION GPS 2002, 24-27 Sept., Portland. pp: $1830-1841$

16 Tiberius C, Kenselaar F. Variance component estimation and precise GPS positioning: case study. J Surv Eng, 2003, 129(1): 11-18

17 He H B, Yang Y X. Real-time estimation of a prior variance-covariance for GPS observations. Acta Geod Cartogr Sin (in Chinese), 2001, 30(1): $42-47$

18 Bona P. Noise characteristics of Trimble 4700 GPS receiver. GPS Nieuwsbrief, 2000, 15(2): 8-11 